\title{
CORRECTIONS
}

\section{Publisher Correction: Women in physics}

\section{Ramin Skibba}

Nature Reviews Physics (2019) https://www.nature.com/articles/s42254-019-0059-x

Published online 7 May 2019

This article has been corrected to clarify that "Berghöfer said" should read "said

Thomas Berghöfer, coordinator of the GENERA project".

https://doi.org/10.1038/s42254-019-0075-x I Published online 28 May 2019 